\title{
A Metamaterial-Inspired Microwave Sensor for Dielectric Characterization of Organic Liquids and Solid Dielectric Substrates
}

\author{
YUNHAO CAO \\ Beihang University \\ Cunjun Ruan ( $\nabla$ ruancunjun@buaa.edu.cn ) \\ Beihang University \\ Kanglong Chen \\ Beihang University \\ Xingyun Zhang \\ Beihang University
}

\section{Research Article}

Keywords: sensor, permittivity, measured, results, samples, proposed, dielectric

Posted Date: August 13th, 2021

DOI: https://doi.org/10.21203/rs.3.rs-798799/v1

License: (c) (i) This work is licensed under a Creative Commons Attribution 4.0 International License. Read Full License 


\title{
A Metamaterial-Inspired Microwave Sensor for Dielectric Characterization of Organic Liquids and Solid Dielectric Substrates
}

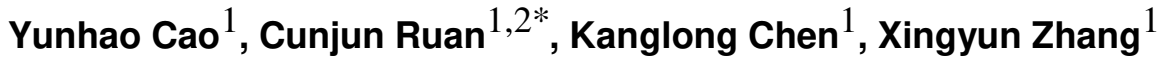 \\ ${ }^{1}$ School of Electronic and Information Engineering, Beihang University, Beijing, 100191, China \\ ${ }^{2}$ Beijing Key Laboratory for Microwave Sensing and Security Applications, Beihang University, Beijing,100191, \\ China \\ *corresponding.ruancunjun@buaa.edu.cn
}

\begin{abstract}
A microwave metamaterial-inspired sensor based on a $13 \times 13$ arrays of Asymmetric Electric Split-Ring Resonator (AESRR) is proposed for dielectric characterization of organic liquids and solid dielectric substrates with low permittivity. The sensor, excited by a pair of patch antennas and working at around $11.575 \mathrm{GHz}$, is fabricated using printed circuit board (PCB) technology. T-shape channel was integrated to the sensor by grooving in the FR-4 substrate which improved the integration and provided the feasibility of liquids detection. Seven liquids and four dielectric substrates are measured by this sensor. The measured results show the transmission frequency shifts from $11.575 \mathrm{GHz}$ to $11.150 \mathrm{GHz}$ as the liquid samples permittivity changes from 1 to 7 and the transmission frequency shifts from $11.575 \mathrm{GHz}$ to $8.260 \mathrm{GHz}$ as the solid substrates permittivity changes from 1 to 9 . The measured results have proven the improved sensitivity and the larger frequency shift $\Delta f$ on material under test (MUTs) compared with the conventional reported sensor. The relative permittivity of liquid samples and solid samples can be fitted by establishing approximate models in CST, respectively. Two transcendental equations derived from measured results are proposed to predict the relative permittivity of liquid samples and solids samples. The accuracy and reliability of measured results and predicted results are numerically verified by comparing them with literature values. The proposed sensor has many advantages, such as low-cost, high-sensitivity, high-robustness, and extensive detecting range, which provided a great potential to be implemented in a lab-on-a-chip sensor system in the future.
\end{abstract}

\section{Introduction}

Metamaterials are artificially made electromagnetic materials composed of sub-wavelength resonant elements, which can manipulate electromagnetic wave beams and exhibit some exotic electromagnetic properties by manipulating their structural geometry and arrangement ${ }^{1,2}$. Microwave sensors have many advantages such as low fabrication and measurement cost, CMOS compatibility, design flexibility, and real-time response, which allow microwave sensors based on metamaterials to be widely used in various fields, such as chemical, biosensing, substrate detection, and microfluidic systems ${ }^{3-12}$.

Recently, many new and improved microwave sensor based on meta-atom structure were proposed. A microfluidic sensor implemented from a single split-ring resonator (SRR) was proposed for the dielectric characterization of liquid samples ${ }^{3}$. A new microwave device which was composed of a microstrip coupled complementary split-ring resonator (CSRR) was proposed in reference 4 as a microfluidic sensor. The sensor can identify water-ethanol mixtures of different concentrations and determine their complex permittivity. A microwave sensor using a Complementary Circular Spiral Resonator (CCSR) was designed for identifying different liquid samples and determining their dielectric constants by dropping the liquids on the sensitive area ${ }^{5}$. Many other microfluidic sensors based on different meta-atom structures ${ }^{6-8}$ were reported to distinguish different liquids and determine their permittivity, such as water, hexane, chloroform, water-ethanol or water-methanol mixtures.

The response of a material to electric signal depends on the permittivity of materials. Thus, many sensors have been proposed and used for material characterization. A microwave sensor, excited by microstrip line and based on the complementary circular spiral resonator (CCSR), was reported for nondestructive evaluation of dielectric substrates ${ }^{9}$. A ring resonator sensor structure was used to identify not only the permittivity but also the thickness of different materials attached to the sensor ${ }^{10}$. And a parabolic equation was proposed to predict the permittivity of material based on the measured resonance frequency. M. S. Boybay et al proposed a microwave method for dielectric characterization of planar materials by using complementary split-ring resonators (CSRRs) working at a $0.8 \mathrm{GHz}-1.3 \mathrm{GHz}$ band $^{11}$. A complementary split-ring resonator (CSRR) sensor, operating at $1.8 \mathrm{GHz}$ to $2.8 \mathrm{GHz}$, was proposed and fabricated for measuring the dielectric constants and loss tangents of materials ${ }^{12}$.

Among the aforementioned microwave characterization devices, all of them are only designed for liquids or solids measurement. And microfluidic sensors ${ }^{4-8}$ can only distinguish some liquids with high permittivity such as water-ethanol mixtures of different concentrations of which the dielectric constants vary greatly. The sensor for material characterization of solids $^{9-12}$ can distinguish different substrate materials with tiny frequency shift $\Delta \boldsymbol{f}$, so there is still a lot of room for improvement in sensitivity. Meanwhile, most of the reported microwave sensors composed by meta-atom structure are easily influenced by the surroundings, leading to the low stability of the sensor. 
In this paper, a microwave metamaterial-inspired sensor based on a $13 \times 13$ arrays of Asymmetric Electric Split-Ring Resonator (AESRR) is proposed. The AESRR metamaterial structure is used in place of electric-SRR (eSRR) structure to provide a novel resonance peak and increase the sensitivity of the sensor. The T-shape channel covering the sensitive region of sensor was integrated to sensor by grooving in the substrate which greatly improved the integration of the microwave passive device. The sensor was fabricated by employing PCB fabrication technology and was verified to have the ability to distinguish seven organic liquids and four common dielectric substrates based on their different frequency shift $\Delta f$. The dielectric constant of MUTs can also be obtained by using relatively accurate simulation models, which were built in CST according to actual measurement environment. Moreover, two transcendental equations are proposed to predict the relative permittivity of liquid samples and solid materials based on the measured resonant frequency, respectively. The proposed sensor can measure not only liquids but also solids and it offers a high robust, high sensitivity, high integration, low fabrication cost and low measurement cost which is promising to be implemented in a lab-on-a-chip system in the future.

On the basis of the previous work ${ }^{27}$, this paper has optimized the substrate thickness and channel for higher sensitivity, wider permittivity range studied and further application. Design of AESRR structure, performance analysis of the whole sensor and sensor fabrication is explained in Section 2. Measurement and transcendental equation for five liquids is performed in Section 3. Measurement and transcendental equation for four solid materials is shown in Section 4. The sensor performance compared to some conventional microwave sensor is discussed in Section 5 and the research is concluded in Section 6.

\section{Sensor design and fabrication}

Metamaterials design and sensor design. Fig. 1(a) shows the schematic of asymmetric eSRR (AESRR) structure, the primary component of the proposed metamaterial-inspired sensor. AESRR is chosen as the fundamental building block of metamaterials because of its simplicity and sensitivity to the change of permittivity environment. The material of AESRR is copper (pure) with electrical conductivity of $5.96 \times 10^{7} \mathrm{~s} / \mathrm{m}$ and the substrate is FR-4 (lossy) with a dielectric constant of 4.4. The dimensions of the AESRR metamaterial structure are shown in Fig. 1(a): gap width $(\mathbf{g})=0.5 \mathrm{~mm}$, line width $(\mathbf{w})=0.75$ $\mathrm{mm}$, the length of the substrate $(\mathbf{p})=10 \mathrm{~mm}$, the thickness of the substrate $(\mathbf{h})=1 \mathrm{~mm}$, the length of the copper $(\mathbf{c})=6 \mathrm{~mm}$, the thickness of the metal part $(\mathbf{t})=0.03 \mathrm{~mm}$, and other parameter: $\mathrm{a}=0.9 \mathrm{~mm}, \mathrm{~b}=3.3 \mathrm{~mm}$, the width of the middle metal arm (d) $=0.9 \mathrm{~mm}$.
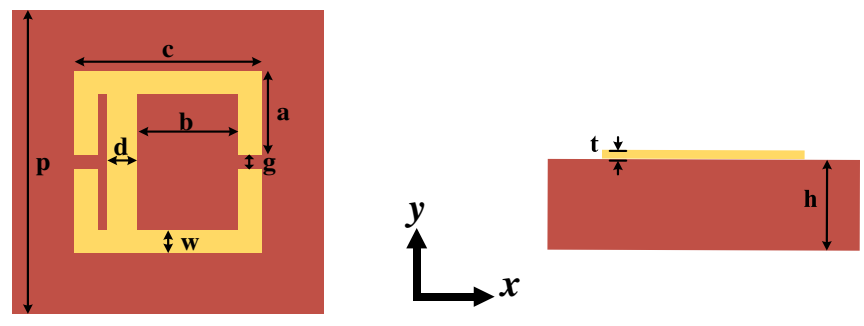

(a)

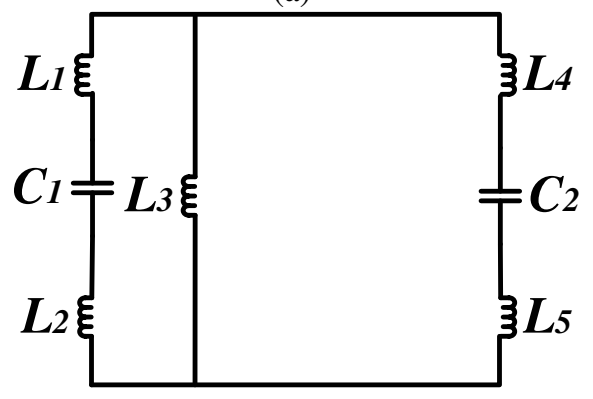

(b)

Figure 1. (a) Schematic of the unit asymmetric eSRR (AESRR) structure. (b) The equivalent circuit of AESRR.

Fig. 1(b) shows the equivalent circuit model of AESRR metamaterial structure. In the equivalent circuit, $L_{1}, L_{2}, L_{3}, L_{4}$, and $L_{5}$ represent the equivalent inductances of the metal arms in the corresponding position, respectively. $C_{1}$ and $C_{2}$ are the equivalent capacitances of the gaps of AESRR. Among the circuit element, the values of inductance $L_{1}-L_{5}$ related to the sensor itself are

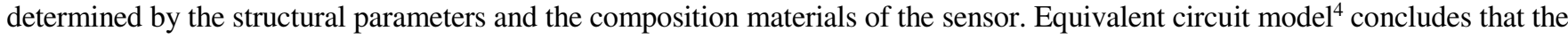
equivalent capacitance of the gaps of sensor is determined by the capacitive effects of sensor itself and the effect of MUTs. According to the equivalent circuit model, the equivalent capacitance $C_{1}$ and $C_{2}$ can be expressed as:

$$
C_{1}=C_{0}{ }^{\prime}+\left(\varepsilon_{\text {sam }} C_{c}\right)^{\prime} \text { and } C_{2}=C_{0}{ }^{\prime \prime}+\left(\varepsilon_{\text {sam }} C_{c}\right)^{\prime \prime}
$$

Where $C_{0}{ }^{\prime}$ and $C_{0}{ }^{\prime \prime}$ model the capacitive effects on both sides of the gaps, which are determined by the dielectric substrate, channels, and surrounding space of the sensor itself. The term $\left(\varepsilon_{s a m} C_{c}\right)^{\prime}$ and $\left(\varepsilon_{s a m} C_{c}\right)^{\prime \prime}$ describe the dielectric contribution from the load MUTs with $C_{C}$ being the capacitance of an empty channel and $\varepsilon_{s a m}$ being the permittivity of MUTs. The value of the effective capacitance $C_{g}$, the total equivalent capacitance of the sensor including $C_{1}$ and $C_{2}$, is influenced by the dielectric materials around the gaps and can be approximately expressed as ${ }^{13}$ :

$$
C_{g}=C_{1}+C_{2}=C_{0}+\varepsilon_{s a m} C_{c}
$$


As mentioned above, $C_{0}$ models the total capacitive effects determined by the sensor itself and the term $\varepsilon_{s a m} C_{c}$ describes the total dielectric contribution from the load MUTs.

The resonant frequency $\left(f_{0}\right)$ of the sensor can be defined as:

$$
f_{0}=\frac{1}{2 \pi \sqrt{L\left(C_{g}\right)}}
$$

Where L represents the total equivalent inductance of the AESRR structure. From (1)-(3), the resonant frequency can be functions of the load MUTs permittivity as (4) shows:

$$
f_{0}=F_{1}\left(\varepsilon_{\text {sam }}\right)
$$

This indicates that the resonant frequency of the sensor will be influenced by the permittivity of the load MUTs ${ }^{14}$. Therefore, the dielectric constant of an unknown MUTs can be determined simply by measuring the different transmission resonance frequencies of sensor due to the interaction with different MUTs.
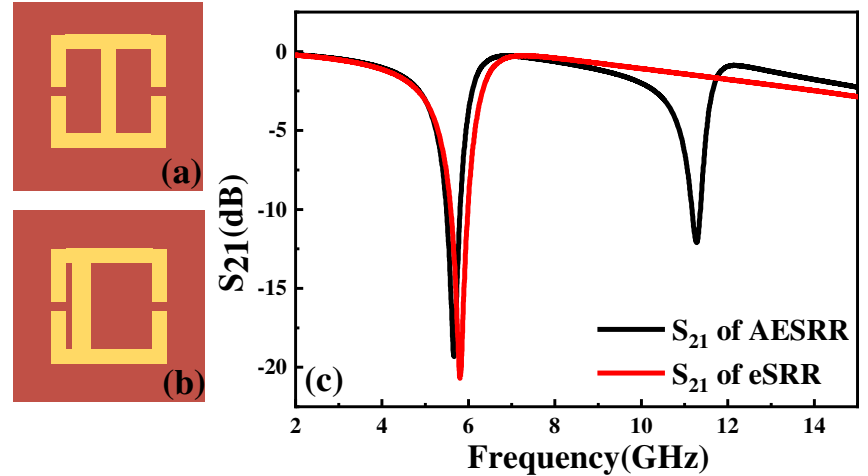

Figure 2. (a) The symmetric eSRR metamaterial structure. (b) The asymmetric eSRR (AESRR) metamaterial structure. (c) Simulated $S_{21}$ of these two structures.

The Fano resonance, discovered by Ugo Fano in 1961, has been described as the interference between continuum of states (the scattered states) and quasi-bound states (resonant states) ${ }^{15}$. V. Sekar et al concluded that introducing Fano resonance to the metamaterial structure is an efficient way to generate a new resonance peak improving the sensitivity of the sensor ${ }^{14}$. The basic eSRR metamaterial structure is shown in Fig. 2(a). To achieve higher sensitivity, the asymmetric eSRR (AESRR) structure is proposed based on the Fano resonance, shown in Fig. 2(b). The Fano resonance is generally caused by asymmetric metamaterial structures ${ }^{14}$. As Fig. 2(c) shows, there appears a novel Fano peak at around $11.30 \mathrm{GHz}$ with the symmetry of eSRR destroyed.

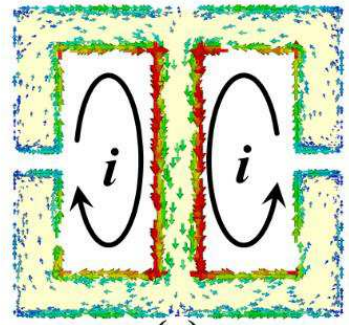

(a)

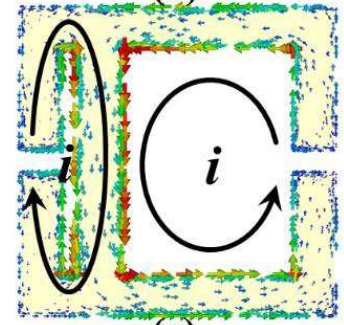

(c)

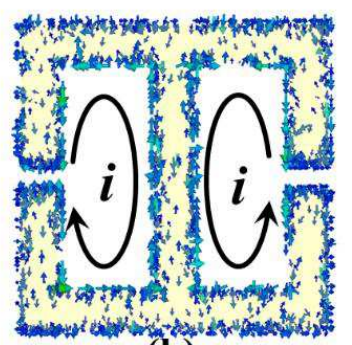

(b)

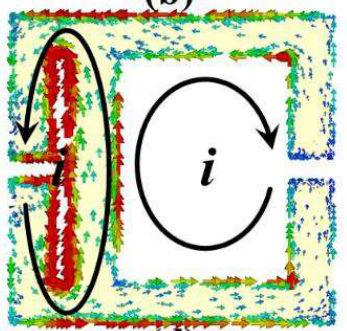

(d)

Min.

Max.

Figure 3. (a) Surface current simulation in eSRR at transmission resonance peak at $5.81 \mathrm{GHz}$. (b) Surface current simulation in eSRR at $11.28 \mathrm{GHz}$. (c) Surface current simulation in AESRR at transmission resonance peak at $5.67 \mathrm{GHz}$. (d) Surface current simulation in AESRR at transmission resonance peak at $11.28 \mathrm{GHz}$. 


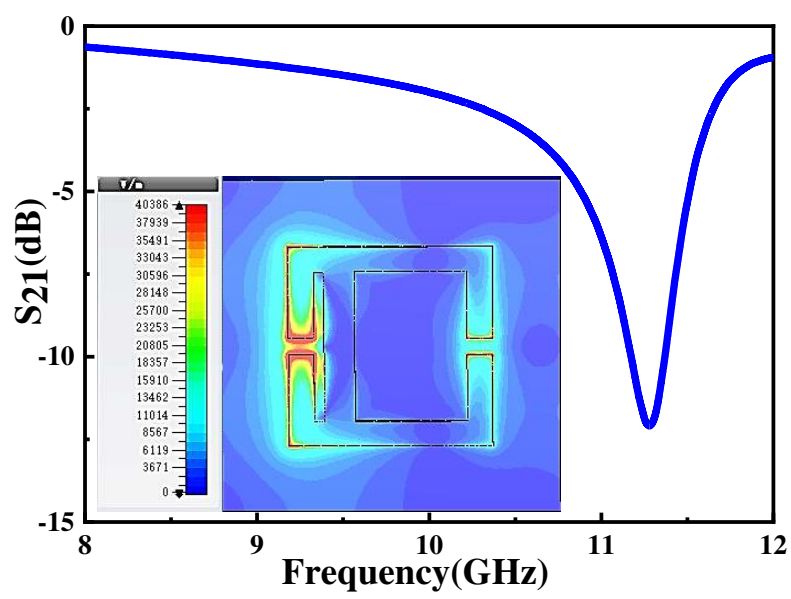

Figure 4. Simulated $S_{21}$ transmission resonance of the proposed sensor without T-shape channel in CST Studio Suite and Electric field distribution at resonance frequency $11.28 \mathrm{GHz}$.

Fig.3 shows surface current simulation in eSRR and AESRR at different frequency. As Fig.3(a) shows, the currents in the two equal metal wire arms of eSRR oscillate in phase and interfere constructively ${ }^{7}$, which generates a resonance peak at $5.81 \mathrm{GHz}$. Compare to the eSRR, the two current loops in AESRR differ with the symmetry broken, leading to a strong coupling between them. Generally speaking, the longer the current path is, the lower the frequency of resonance peak is; The shorter the current path, the higher the resonant frequency. In Fig.3(c), The right current loop is slightly stronger than the left current loop. In Fig.3(d), The left current loop is obviously stronger than the left current loop. The resonant peak of AESRR at $5.67 \mathrm{GHz}$ is from the large current path on the right and the resonant peak of AESRR at $11.28 \mathrm{GHz}$ is from the small current path on the right. By comparing Fig.3(b) and Fig.3(d), the current loop in AESRR is stronger and the current difference between the two loops in AESRR is larger, which created a strong coupling and generated a new resonance peak at $11.28 \mathrm{GHz}$. And the electric field distribution of AESRR at transmission resonance peak at $11.28 \mathrm{GHz}$ is shown in Fig.4. Electric field distribution embedded in Fig. 4 tells us that a strong electric field establishes between gaps, especially the left one. To ensure the performance of the sensor, the channel should cover the sensitive areas. Whereas the width of the gap (g) increases the difficulty of the microfluidic channel processing and integration. Considering the integration difficulty and processing cost, finally, we decide to process and integrate the T-shape microfluidic by grooving in the FR-4 substrate as Fig. 5 shows. Another consideration was a lab-on-chip system implementation, which is convenient with microfluidic channel in substrate.

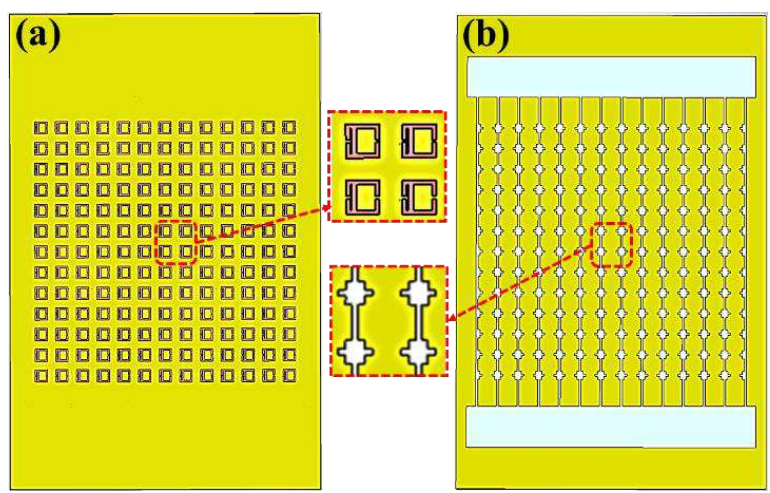

Figure 5. (a) the $13 \times 13$ periodic arrays AESRR structure of the integrated AESRR metamaterial-inspired sensor. (b) the schematic of the whole microfluidic channel.

As Fig. 5 shows, a metamaterial-inspired sensor based on a 13×13 AESRR arrays structure has been designed to enable the feasibility and the accuracy of the measured results. Fig. 5(a) shows a 13×13 AESRR arrays which is large enough to cover the radiation range of the antenna to ensure the reliability of the measurement. Fig. 5(b) is the schematic of the whole microfluidic channel. As Fig. 5(b) shows, we also designed two square grooves on the both edges of the microfluidic channel so that it is convenient for us to make the liquid samples in the square grooves fill in the channel with the help of the gravity and fluidity of liquid samples.

In order to verify the performance of the proposed sensor and compare the sensitivity of different resonance peaks, sensors based on different metamaterial structures was analyzed in the CST [25-26]. By changing the dielectric constant of MUTs in the channel, different resonance peaks have different frequency shift $|\Delta f|$. Fig. 6 clearly illustrates that the sensitivity of the peak of AESRR is much better than that of the other two resonance peaks. The simulated frequency shift $|\Delta f|$ shows that the resonance peak of AESRR at around $6 \mathrm{GHz}$ and the resonance peak of eSRR at around $6 \mathrm{GHz}$ are insensitive to small changes in the dielectric environment unless the changes in the dielectric environment are large enough. At the same time, the resonant peak of AESRR at around $11 \mathrm{GHz}$ has a large $|\Delta f|$ even for the slight changes of dielectric environment. Based on the simulated results, the resonance 
peak at around $11 \mathrm{GHz}$ was selected for measuring different MUTs with slight dielectric change.

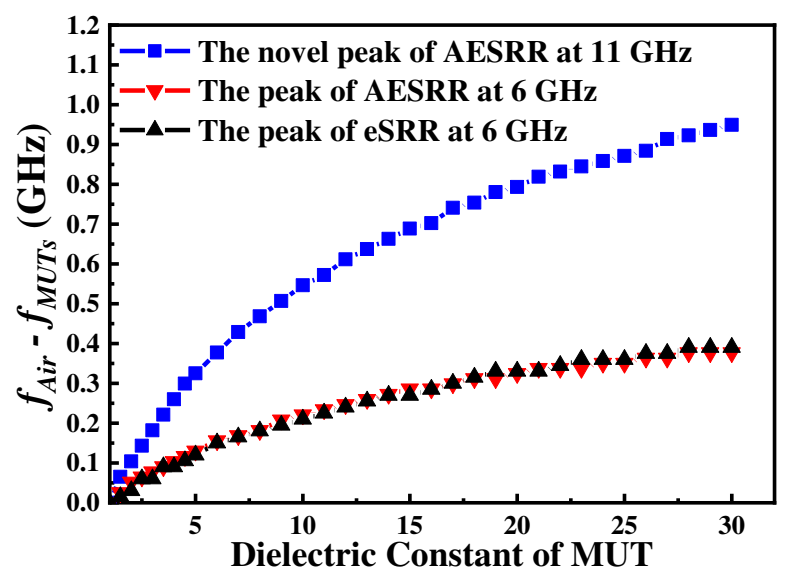

Figure 6. Simulated results of different resonance peaks (the peaks of AESRR at around $6 \mathrm{GHz}$ and $11 \mathrm{GHz}$; the peaks of eSRR at around $6 \mathrm{GHz}$ ) with channel filled with different dielectric materials.

Sensor fabrication and measurement setup. We fabricated the sensor based on the AESRR by employing the PCB fabrication technology. Considering the characteristic of the patch antenna and in order to ensure the accuracy of the measurement result, a $13 \times 13$ AESRR arrays plant was fabricated on the FR-4 substrate, with a relative permittivity of 4.4 and was $13 \mathrm{~cm} \times 25 \mathrm{~cm}$ in size, shown in Fig. 7. This sensor is a kind of passive microwave device, and has the advantage being high-robust, reusable, real-time and high-sensitivity.
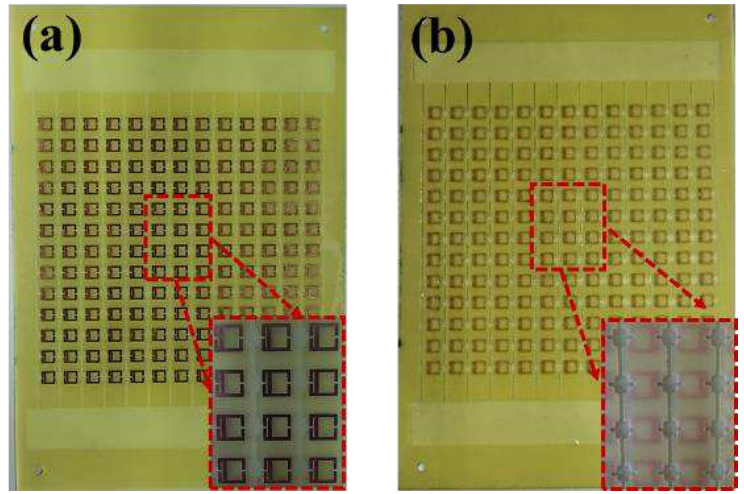

Figure 7. (a) Overall photograph and partial enlarge view of the $13 \times 13$ periodic arrays AESRR structure. (b) Overall photograph and partial enlarge view of the whole microfluidic channel.

In the simulation software (CST) the distance between transmitting antenna and sensor must greater than $10.2 \mathrm{~mm}$ which is determined by substrate thickness $(1 \mathrm{~mm})$ and periodic structure characteristics. Fig. 8 shows the effect of the distance on the measured results. It's not hard to find the distance between transmitting antenna and sensor has very little effect on the measured results. Considering the attenuation of the antenna is severe when the distance is large, so we decided to keep the distance between $1.2 \mathrm{~mm}$ and $1.6 \mathrm{~mm}$.

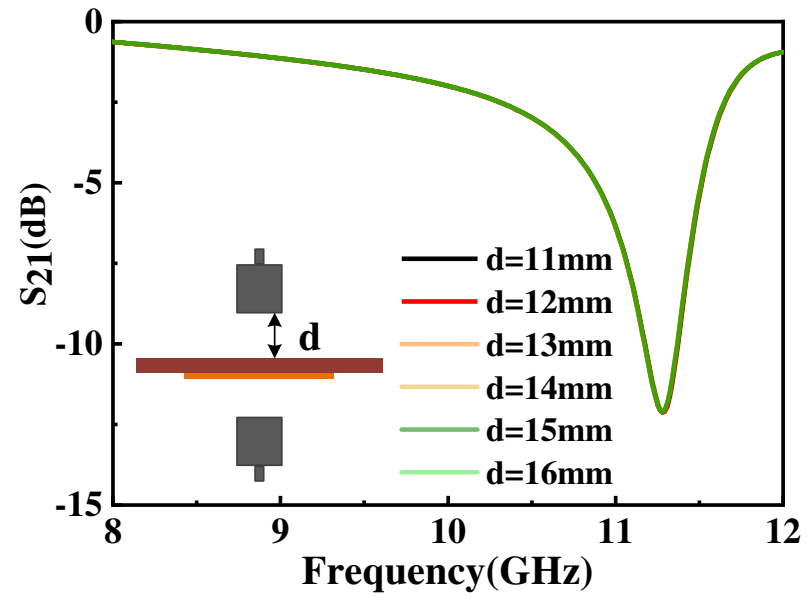

Figure 8. Simulation results of the influence of the distance between the antenna and the sensor on the measured results. 
The schematic diagram of the developed microwave sensor for dielectric characterization and its deployment are shown in Fig. 9. All the experiments were carried out at a room temperature of $25^{\circ} \mathrm{C}$. In our measurement, signal is generated by vector network analyzer (AV3672C, $10 \mathrm{MHz}-43.5 \mathrm{GHz}$ ), and a pair of patch antennas are used to transmit and receive signals, shown in Fig. 9.

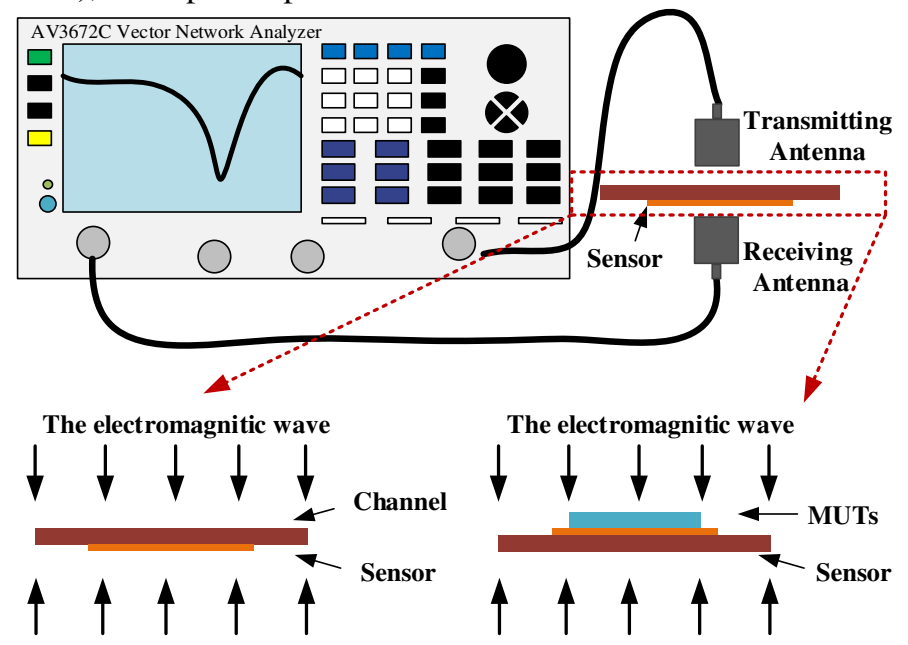

Figure 9. Deployment of the proposed microwave sensing system for the dielectric characterization of organic liquids and solid dielectric substrates with low dielectric constant.

Device Characterization. The measured and simulated results of $S_{21}$ transmission coefficient of the sensor is shown in Fig. 10. It's obvious that the measured results are basically consistent with the simulation results. When the measurement platform and the sensor without MUT was set up according to the deployment in Fig. 9, about 15 times measurement was carried out to make sure the measured results are reliable, and the measured results indicate that the sensitive peak is stabilized at $11.575 \mathrm{GHz}$. Detailed data of the simulated and measured results are given in Table 1. The difference of amplitude between simulated and measured results is mainly due to the characteristics of patch antenna, fabrication tolerance, conductor, dielectric and radiation losses. Considering that the proposed device distinguishes different MUTs based on the shift of the resonant frequency, the measured results indicate that the device conforms to the design and can be used as a sensor.

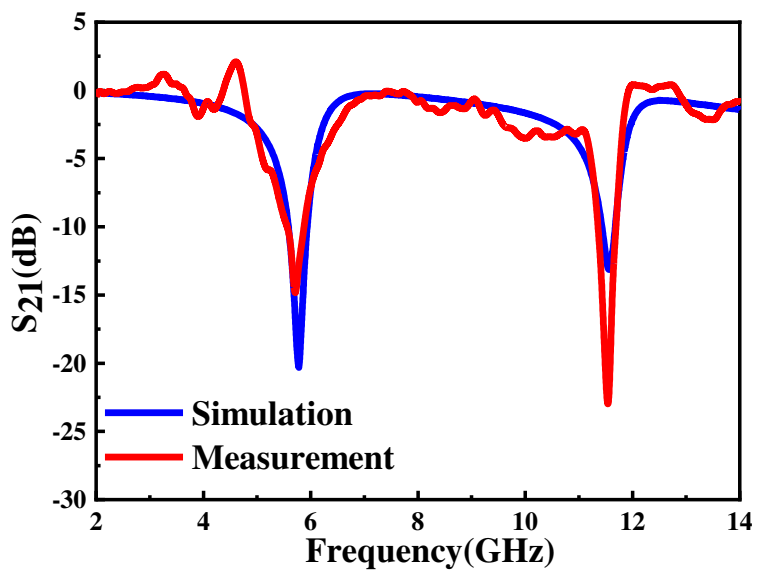

Figure 10. Simulated and measured transmission response $S_{21}$ of the sensor without MUTs.

\begin{tabular}{ccccc}
\hline \hline Results & $f_{1}(\mathrm{GHz})$ & ${\text { Notch } \text { Depth }_{1}}$ & $f_{2}(\mathrm{GHz})$ & ${\text { Notch } \text { Depth }_{2}}$ \\
\hline Simulated & 5.760 & $-20.2 \mathrm{~dB}$ & 11.570 & $-13.2 \mathrm{~dB}$ \\
Measured & 5.720 & $-16.1 \mathrm{~dB}$ & $11.575 \pm 0.01$ & $-25.4 \mathrm{~dB}$ \\
\hline \hline
\end{tabular}

Table 1. Measured and simulated results of the microwave sensor without MUTs

\section{Measured results of different liquids with low dielectric constant}

The resonant peak at around $11.60 \mathrm{GHz}$ is sensitive to the small change of dielectric environment, so we try to measure different liquids with low dielectric constant to verify the performance of the sensor. Different organic liquids that have a homogeneous dielectric distribution and high fluidity, such as peanut oil (LuHua), corn oil (Longevity Flower), sunflower seed oil (Longevity Flower) soybean oil (Golden dragon fish), IPA (DongWu), Ethyl acetate (DongWu), and ethanol (Aladdin) were chosen as MUTs. In order to minimize the impact of contamination and humidity from the previous test sample liquids, we washed the 
channel with detergent and brush firstly, then rinsed the channel repeatedly with alcohol solution and dried the remaining alcohol with a small hair dryer. Finally, after the sensor was laid flat for about 30s to ensure that the alcohol evaporates adequately, the next liquid sample was dropped in the channels. When measuring the volatile liquid samples, we record the measured data quickly. Fig.11 shows the overall experiment platform for measuring different liquid samples. Each sample was measured about 15 times to ensure the reliability of the measured results. The measured results $S_{21}$ of different liquids sample are presented in Fig. 12 and the specific measured data is shown in Table.2. The carve of air is regarded as a reference signal, and the other liquids curves with different resonant frequency are measured transmission coefficient $S_{21}$. It's obvious that there are different resonant frequencies when the channel with different samples, so the proposed sensor can be used for identifying different liquids with low permittivity.

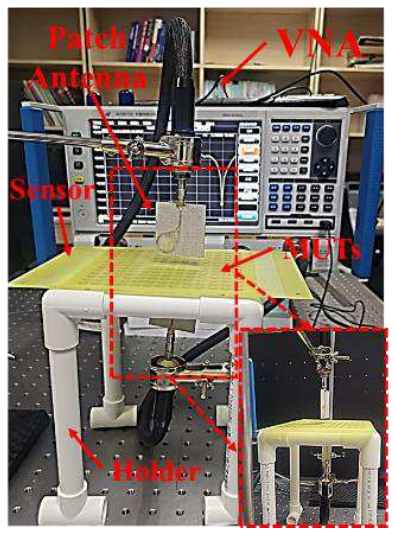

Figure 11. Photograph of the experiment platform for measuring different liquid samples.

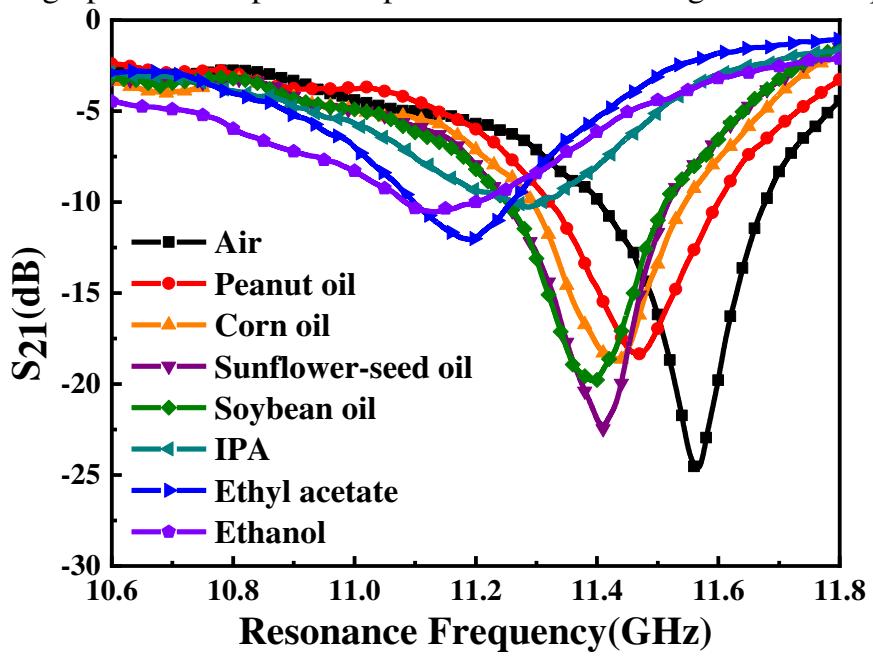

Figure 12. Measured transmission coefficient $S_{21}(\mathrm{~dB})$ of sensor due to interaction with different organic liquids. Resonance frequencies of sensors due to interaction with Air, Peanut oil, Corn oil, Sunflower-seed oil, Soybean oil, IPA, Ethyl acetate, Ethanol are $11.575 \mathrm{GHz}, 11.470 \mathrm{GHz}, 11.420 \mathrm{GHz}, 11.410 \mathrm{GHz}, 11.390 \mathrm{GHz}, 11.287 \mathrm{GHz}, 11.200 \mathrm{GHz}$ and $11.150 \mathrm{GHz}$, respectively.

\begin{tabular}{cccc}
\hline \hline Liquid Samples & Literature $\varepsilon_{r}$ & Resonance Frequency (GHz) & $\begin{array}{c}\text { Notch Depth } \\
(\mathrm{dB})\end{array}$ \\
\hline Ethanol & 7.02 & 11.150 & -10.5 \\
Ethyl acetate & 6.04 & 11.200 & -12.0 \\
IPA & 4.38 & 11.287 & -10.3 \\
Soybean oil & 2.99 & 11.390 & -19.6 \\
Sunflower seed oil & 2.75 & 11.410 & -22.5 \\
Corn oil & 2.63 & 11.420 & -18.6 \\
Peanut oil & 2.01 & 11.470 & -18.4 \\
Air & 1.00 & 11.575 & -24.7 \\
\hline \hline
\end{tabular}

Table 2. Measured results of the microwave sensor with different organic liquids

Fitting model of sensor with different liquids. The measured results show that different liquid samples with low dielectric constant can be distinguished by the sensor. The resonance frequency and amplitude of the resonant peak can be 
separately used for calculating the real part and the imaginary part of the unknown liquids' permittivity ${ }^{15}$. Considering that the attenuation of the patch antenna has a great impact on the measured amplitude, so we only can analyze the real part of the sample liquids' permittivity. According to the actual situation of the liquids in the measurement, a relatively accurate model, which includes the microfluidic channel part, is built and shown in Fig. 13. The blue part of the model is the channel filled with different liquids. The geometrical parameters of T-shape channel shown in Fig. 13(a) are as follows: $d_{1}=0.5 \mathrm{~mm}, d_{2}=d_{3}=d_{4}$ $=1 \mathrm{~mm}, d_{5}=4 \mathrm{~mm}$, the depth of the T-shape channel $\left(d_{6}\right)$ is $0.5 \mathrm{~mm}$. By changing the dielectric constant of the liquids in the model, making the simulated resonance frequency fit the measured results as much as possible, then we can get a fitted permittivity of the liquid and the obtained value is very close to the real dielectric constant of the liquid.
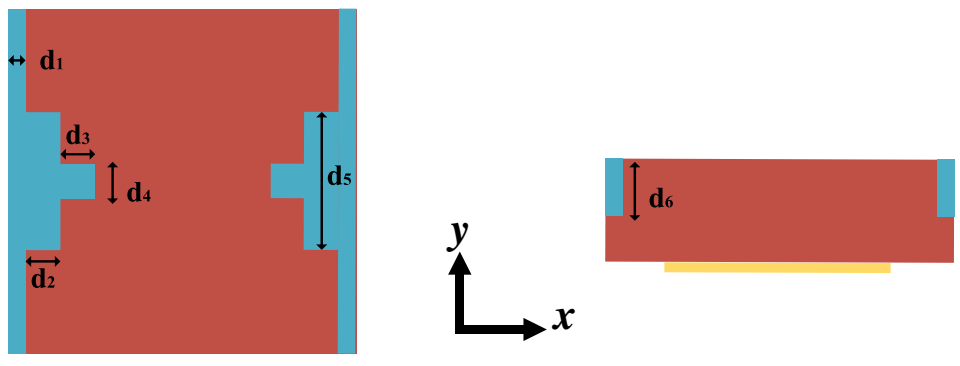

Figure 13. Simulation model of the proposed microwave sensor using CST with the sample liquids filling with the channels.

The Model between the dielectric constant and resonant frequency for liquids with low permittivity. Using the fitting model mentioned above, the relative permittivity of liquids can be obtained and the comparison of measured results and simulated results are shown in Fig. 14. The difference of amplitude is mainly caused by the fabrication tolerance, conductor, dielectric and radiation losses. The simulated and fitted dielectric constants of different organic liquid samples are tabulated in Table 3. As Fig. 15 shows, the measured $\varepsilon_{r}$ of peanut oil, corn oil, sunflower oil, soybean oil, IPA, ethyl acetate, and ethanol match well with those measured in the literature ${ }^{5,7,16-20}$, which indicates the reliability and accuracy of the measured results and the simulation model.
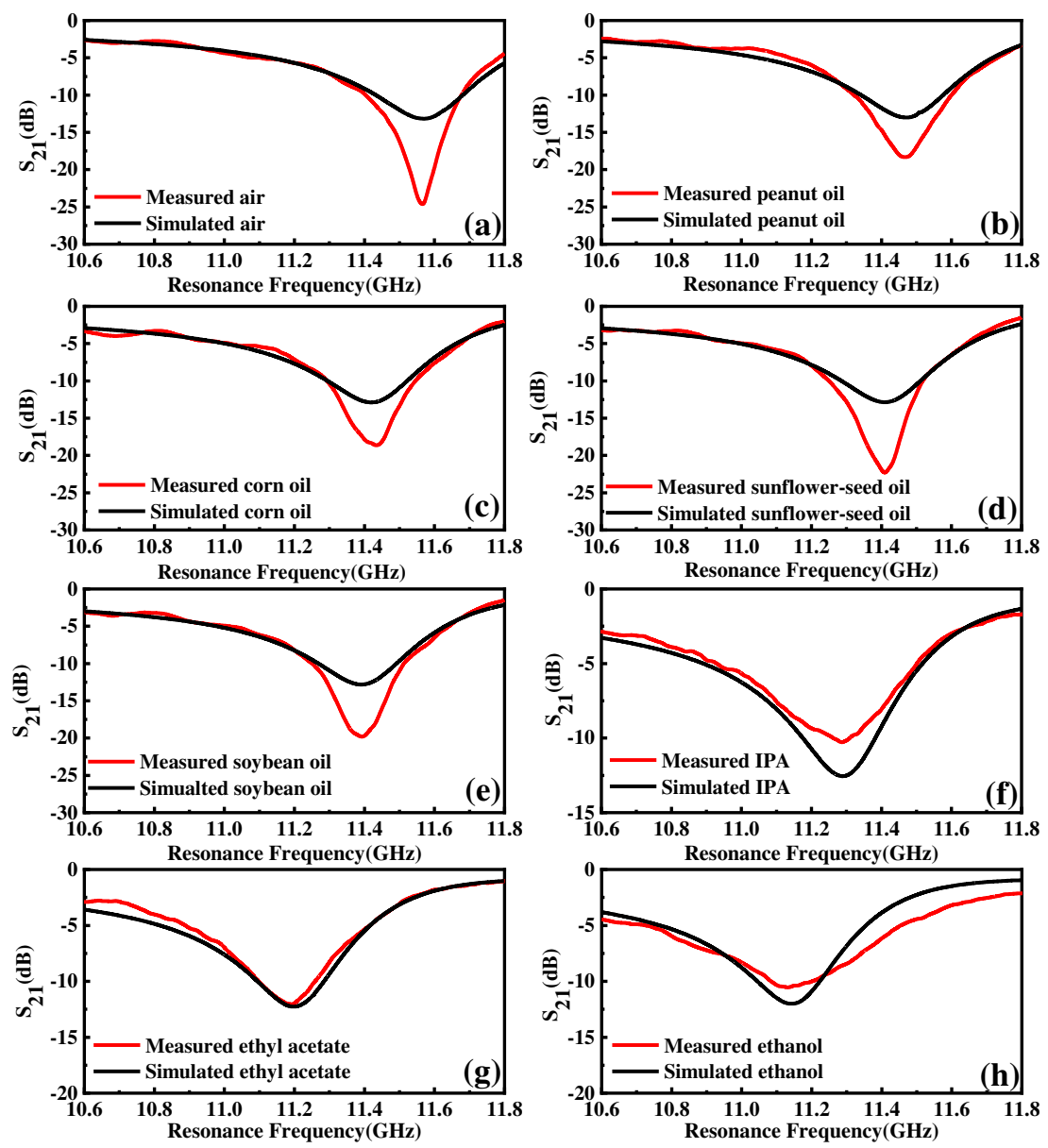

Figure 14. Simulated and measured resonance frequency of (a) Air (reference). (b) Peanut oil. (c) Corn oil. (d) Sunflowerseed oil. (e) Soybean oil. (f) IPA. (g) Ethyl acetate. (h) Ethanol. 


\begin{tabular}{cccccc}
\hline \hline Liquid Samples & Measured $f(\mathrm{GHz})$ & $\begin{array}{c}\text { Simulated } f \\
(\mathrm{GHz})\end{array}$ & Literature $\varepsilon_{r}$ & Fitted $\varepsilon_{r}$ & Fitted Error (\%) \\
\hline Ethanol & 11.150 & 11.140 & 7.02 & 7.00 & $0.28 \%$ \\
Ethyl acetate & 11.200 & 11.198 & 6.04 & 6.00 & $0.66 \%$ \\
IPA & 11.287 & 11.288 & 4.38 & 4.38 & $0.00 \%$ \\
Soybean oil & 11.390 & 11.390 & 2.99 & 2.90 & $3.01 \%$ \\
Sunflower-seed oil & 11.410 & 11.410 & 2.75 & 2.65 & $3.63 \%$ \\
Corn oil & 11.420 & 11.420 & 2.63 & 2.55 & $3.04 \%$ \\
Peanut oil & 11.470 & 11.470 & 2.01 & 1.97 & $2.03 \%$ \\
Air & 11.575 & 11.568 & 1.00 & 1.00 & $0.00 \%$ \\
\hline \hline
\end{tabular}

Table 3. Measured and simulated results of the microwave sensor with different organic liquids

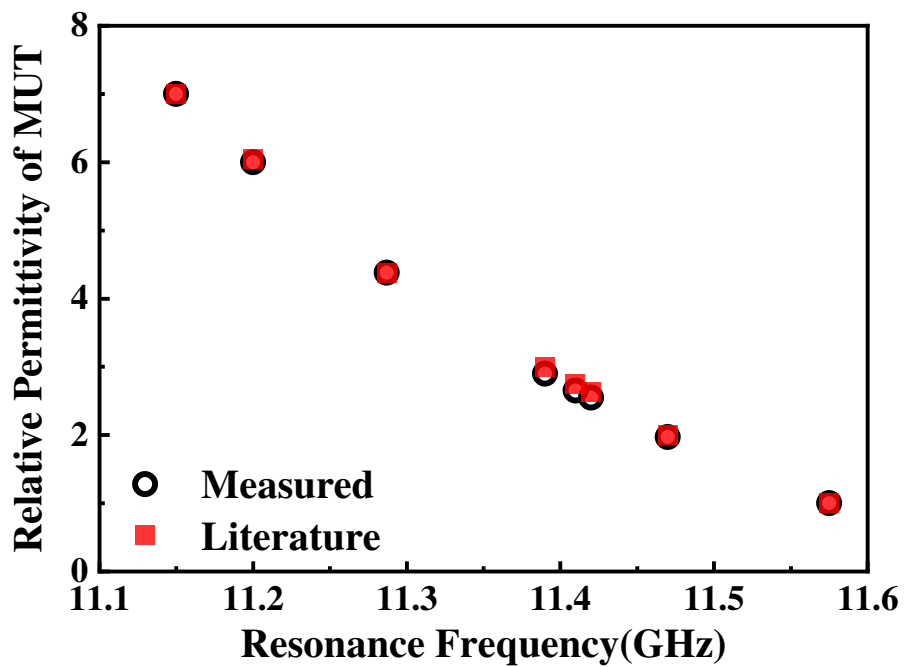

Figure 15. Comparison between the literature values of the relative permittivity from ${ }^{5,14,16,17-21,25}$ and the ones measured by using the designed sensor.

The model between the dielectric constant and resonant frequency for liquids with low permittivity. G. GalindoRomera et al proposed a parabolic equation ${ }^{16}$ between resonance frequency $\mathrm{f}$ and dielectric constants $\varepsilon_{r}$ which can be used to estimate the relative permittivity of some other unknown liquids. The parabolic equation with three constant parameters is as follows:

$$
f_{\text {r.MUT }}=A_{1}+A_{2} \varepsilon_{r}^{\prime}+A_{3} \varepsilon_{r}^{\prime 2}
$$

Here, $\varepsilon_{r}^{\prime}$ is the relative permittivity of liquid sample. $A_{1}, A_{2}$, and $A_{3}$ are constant values. The reference MUT is air whose dielectric constant is 1 . Considering that $f_{r . A i r}$, the resonant frequency of sensor with empty channel, is a constant value. Based on reference ${ }^{15}$, equation (5) can be expanded with respect to $\left(\varepsilon_{r}^{\prime}-1\right)$, as (6) shows:

$$
f_{\text {r.MUT }}=11.575+A_{2}\left(\varepsilon_{r}^{\prime}-1\right)+A_{3}\left(\varepsilon_{r}^{\prime}-1\right)^{2}
$$

Based on the measured results of different liquids, the constant parameters $A_{1}, A_{2}$,and $A_{3}$ of (6) can be determined. The final parabolic equation (6) becomes

$$
f_{r . M U T}=11.575-0.10863\left(\varepsilon_{r}^{\prime}-1\right)+0.00646\left(\varepsilon_{r}^{\prime}-1\right)^{2}
$$

The curve of the fitting parabolic equation (7) is shown in Fig. 16. To calculate the relative permittivity of unknown liquids, the transcendental equation can be expressed as:

$$
\varepsilon_{r}^{\prime}=\frac{0.10863-\sqrt{0.0118-0.2584\left(11.575-f_{r . M U T}\right)}}{0.01292}+1
$$

Based on the measured resonance frequency, the transcendental equation can be used to estimate relative permittivity of unknown liquids with permittivity ranges from 1 to 9 . To verify the reliability of the transcendental equation, measured resonance frequency are used to estimate the dielectric constant of different liquid samples are compared in Fig. 16 and Table 4. And the model error shows the reliability of the transcendental equation (8). 


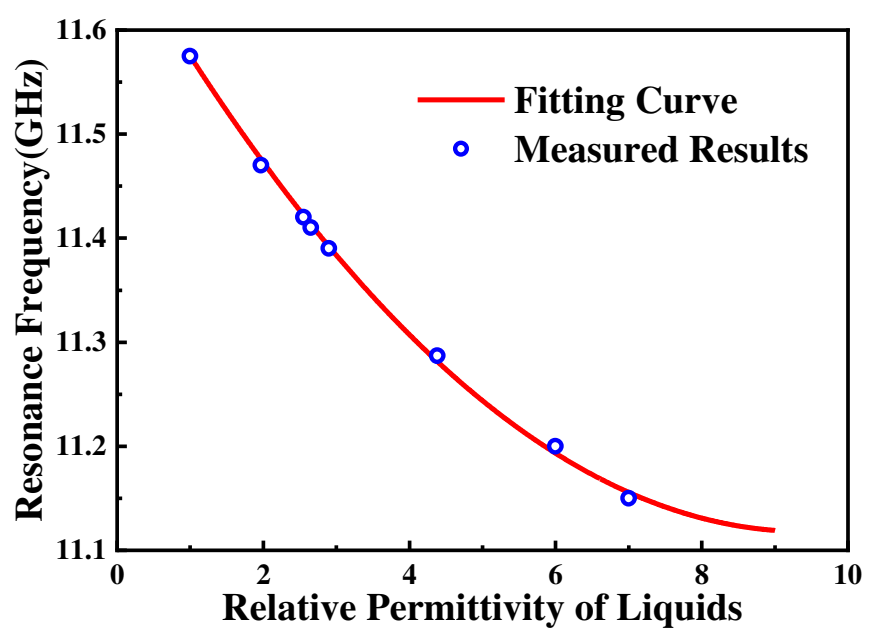

Figure 16. The carve of the fitting parabolic equation (7) based on measured results.

\begin{tabular}{cccc}
\hline \hline Liquid Samples & Measured $\varepsilon_{r}$ & Calculated $\varepsilon_{r}$ & Model Error \\
\hline Ethanol & 7.00 & 7.19 & +0.19 \\
Ethyl acetate & 6.00 & 5.86 & -0.14 \\
IPA & 4.38 & 4.30 & -0.08 \\
Soybean oil & 2.90 & 2.92 & +0.02 \\
Sunflower seed oil & 2.65 & 2.69 & +0.04 \\
Corn oil & 2.55 & 2.57 & +0.02 \\
Peanut oil & 1.97 & 2.02 & +0.05 \\
Air & 1.00 & 1.00 & +0.00 \\
\hline \hline
\end{tabular}

Table 4. Dielectric constant calculated using measured resonance frequency and transcendental equation (8)

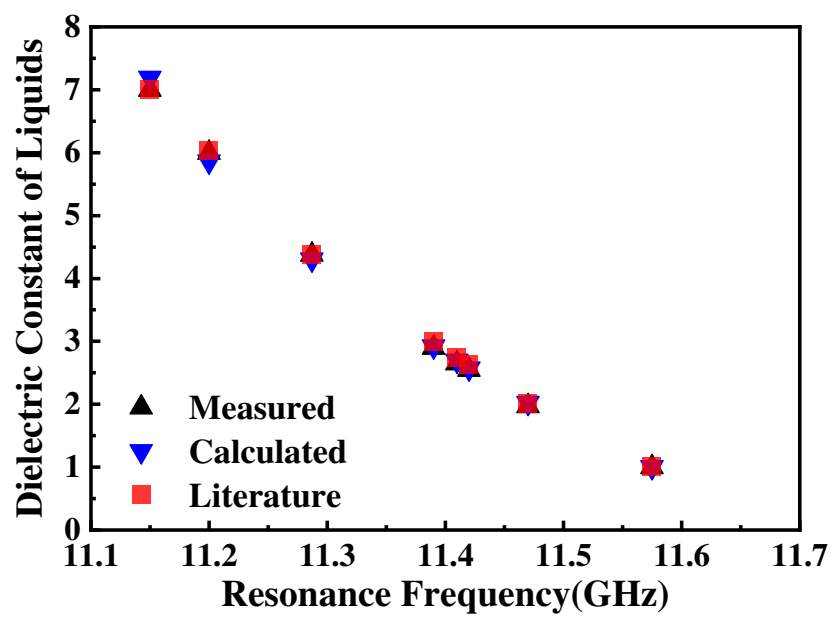

Figure 17. Comparison between the measured, calculated and literature values of dielectric constant for different liquids.

Fig. 17 shows that the calculated and measured dielectric constants of different liquid samples measured in this paper agree well with those reported in the literature ${ }^{5,7,16-20}$, which indicates the reliability and accuracy of the transcendental equation to a certain extent. The estimated and measured values of samples' dielectric constant are a little lower than the literature values, mainly for the reason that the dielectric constant of liquid will decrease with the increase of frequency ${ }^{21}$.

\section{Measurement for solid dielectric substrates}

Simulation and measurement of common solid dielectric materials. Considering the actual measurement situation of the solids, a simulation model including air layers was built in CST. In the actual measurement, the slight bending of the MUTs resulted in the fact that the MUTs and the sensor did not fit tightly, so we added the air layer 2 to the model shown in Fig. 18 to ensure the accuracy of simulation results ${ }^{12}$. The thickness of air layer 1, air layer 2 and MUT are $0.03 \mathrm{~mm}, 0.02 \mathrm{~mm}$ and $1 \mathrm{~mm}$, respectively. Common solid dielectric materials (Teflon, Quartz, FR-4, Ceramics) were simulated and the simulated results is shown in Fig. 19. The simulated results show that the sensor has the ability to distinguish different solid materials 
with high sensitivity and large frequency shift $\Delta f$.

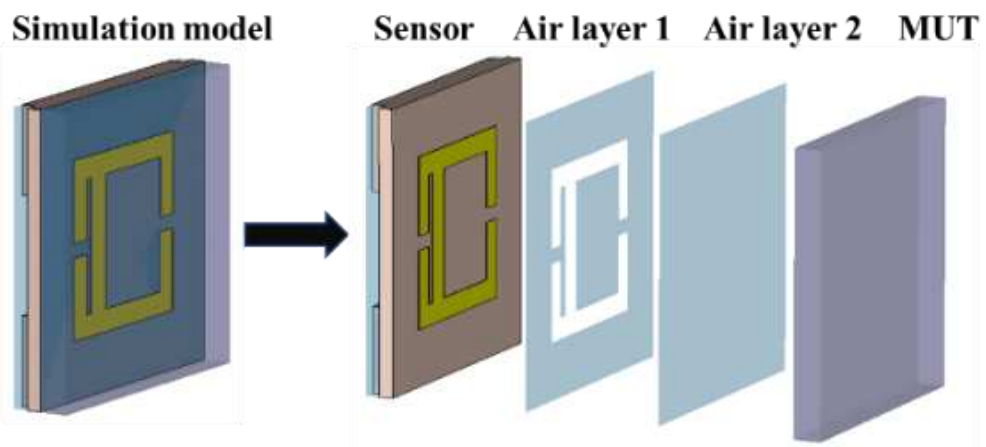

Figure 18. Simulation model and its profile chart which includes the air layer.

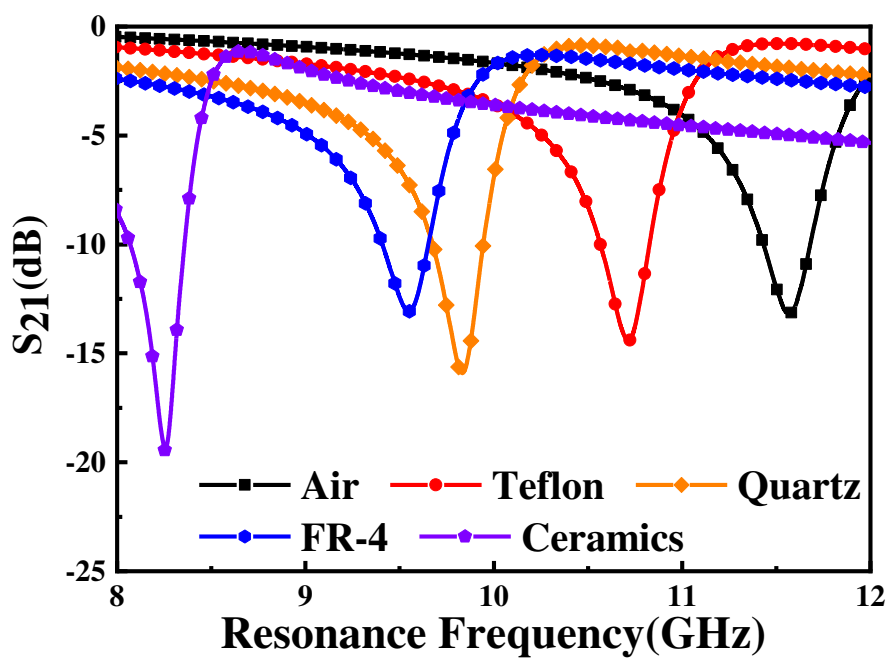

Figure 19. Simulated transmission coefficient $S_{21}(\mathrm{~dB})$ of sensor due to interaction with different MUTs. Resonance frequencies of sensors due to interaction with Air, Teflon, Quartz, FR-4, and Ceramics are $11.57 \mathrm{GHz}, 10.71 \mathrm{GHz}, 9.84 \mathrm{GHz}$, $9.55 \mathrm{GHz}$, and $8.25 \mathrm{GHz}$, respectively.

As Fig. 20 shows, a measurement platform was set up according to the schematic diagram of Fig. 9. The measured results of sensor with different MUTs were shown in Fig. 21 which are basically consistent with the simulated results.

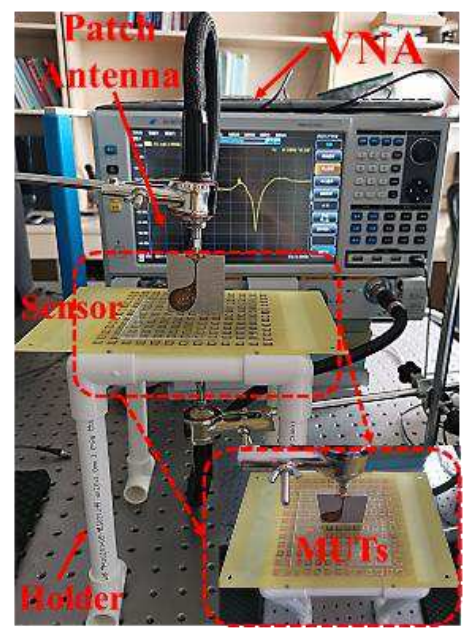

Figure 20. Photograph of the experiment platform for measuring different MUTs. 


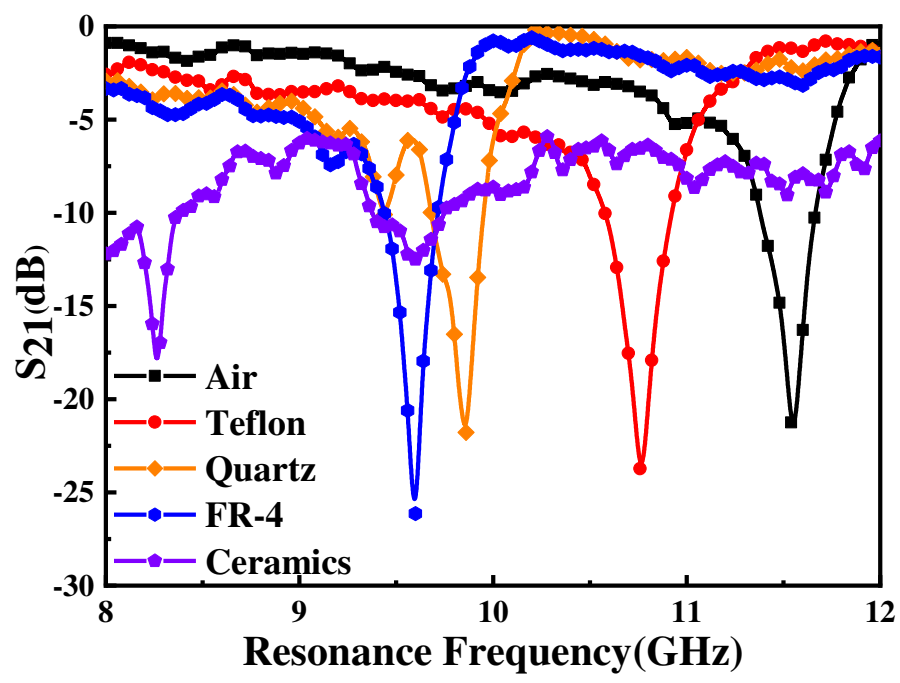

Figure 21. Measured transmission coefficient $S_{21}(\mathrm{~dB})$ of sensor due to interaction with different MUTs. Resonance frequencies of sensors due to interaction with Air, Teflon, Quartz, FR-4, and Ceramics are $11.575 \mathrm{GHz}, 10.760 \mathrm{GHz}, 9.860$ $\mathrm{GHz}, 9.600 \mathrm{GHz}$, and $8.260 \mathrm{GHz}$, respectively.

Analysis of the measured results. The relative permittivity of Air, Teflon, Quartz, FR-4, and Ceramics are well known and shown in Table 4. To verify the accuracy of the simulation model and the measured results, the comparison between simulated and measured results is shown in Fig. 22 and Table 5. The differences between simulated and measured resonance frequency are very small and can be attributed to fabrication tolerance and measurement errors. And the differences between simulated and measured amplitude are mainly caused by the fabrication tolerance, conductor, dielectric and radiation losses. The irregularity of the measured carve is mainly caused by the heterogeneity of the MUTs and dielectric and radiation losses. Fig. 23 shows that the measured relative permittivity of MUTs match well with the literature values reported in references ${ }^{9-12}$, which indicates the accuracy of the measured results and the reliability of the sensor proposed in this paper.
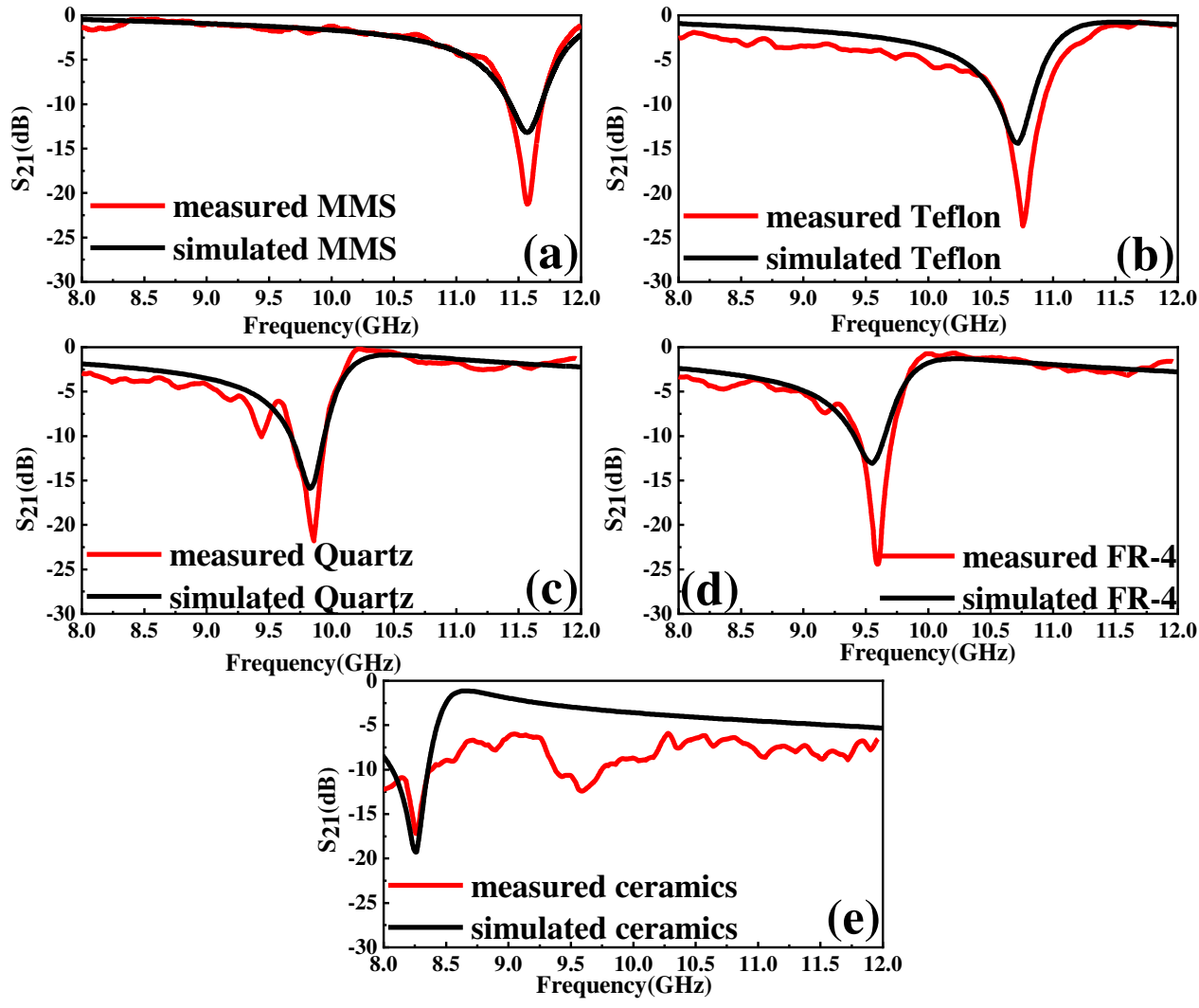

Figure 22. Comparison between simulated and measured transmission coefficient $S_{21}$ (dB) of (a) Air (reference). (b) Teflon.

(c) Quartz. (d) FR-4. (e) Ceramics. 


\begin{tabular}{ccccc}
\hline \hline MUTs & $\varepsilon$ & Simulated $f(\mathrm{GHz})$ & Measured $f(\mathrm{GHz})$ & $\begin{array}{c}\text { Difference } \\
(\mathrm{GHz})\end{array}$ \\
\hline Air & 1 & 11.570 & 11.575 & 0.005 \\
Teflon & 2.1 & 10.710 & 10.760 & 0.050 \\
Quartz & 3.75 & 9.840 & 9.860 & 0.020 \\
FR-4 & 4.3 & 9.550 & 9.600 & 0.050 \\
Ceramics & 9.0 & 8.250 & 8.260 & 0.010 \\
\hline \hline
\end{tabular}

Table 5. Comparison between simulated and measured results of sensors for different MUTs

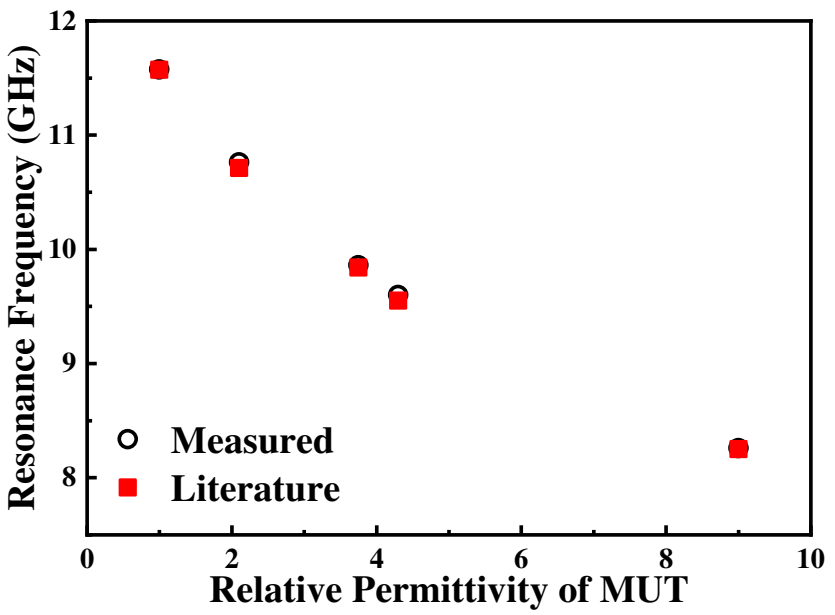

Figure 23. Comparison between the literature values of the relative permittivity from [9-12] and the ones measured using the design sensor.

The model between the dielectric constant and resonant frequency for solids with low permittivity. In this paper, the fitting equation for the whole sensor are formulated with fitting parameters using measured results. The resonant frequencies of the sensor very when material under test (MUT) is placed on the sensor ${ }^{22}$. The variation in resonant frequency can be expressed by the following equation:

$$
f_{r . M U T}=f_{r . A i r} \sqrt{\frac{\varepsilon_{e f f, A i r}}{\varepsilon_{e f f, M U T}}}
$$

where $f_{r . M U T}$ and $f_{r . A i r}$ are resonance frequencies of sensor with and without MUT, respectively. And $\varepsilon_{e f f, A i r}$ and $\varepsilon_{e f f, M U T}$ are effective permittivity of air and MUTs, respectively. Figure 10 shows the relationship between relative permittivity of MUT and the resonant frequency of sensor due to interaction with MUT. And the relationship shows that the resonance frequency is decreasing by the increasing the relative permittivity of MUT. In reference ${ }^{22}$, a parabolic equation between relative permittivity of MUTs and the resonant frequency of sensor is established. It's shown in the following equation:

$$
f_{r . M U T}=A_{1}+A_{2} \varepsilon_{r}^{\prime}+A_{3} \varepsilon_{r}^{\prime 2}
$$

Here, $\varepsilon_{r}^{\prime}$ is relative permittivity of MUT. $A_{1}, A_{2}$, and $A_{3}$ are constant values. The reference MUT is air whose dielectric constant is 1 . Considering that the resonant frequency of sensor without MUT $f_{r . A i r}$ is a constant value and based on reference ${ }^{15}$, equation (10) can be expanded with respect to $\left(\varepsilon_{r}^{\prime}-1\right)$, as equation (11) shows:

$$
f_{r . M U T}=A_{1}+A_{2}\left(\varepsilon_{r}^{\prime}-1\right)+A_{3}\left(\varepsilon_{r}^{\prime}-1\right)^{2}
$$

Based on the measured results of materials (Air, Teflon, Quartz and Ceramics), the constant parameters $A_{1}, A_{2}$, and $A_{3}$ of (11) can be determined. Then, equation (11) becomes:

$$
f_{r . M U T}=11.575-0.74629\left(\varepsilon_{r}^{\prime}-1\right)+0.04152\left(\varepsilon_{r}^{\prime}-1\right)^{2}
$$

Materials (FR-4) are stand dielectric substrate for which dielectric constant is well known. We used (12), fitted based on the measured results of other MUTs, to estimate the relative permittivity of FR-4 to test the reliability of this model. The $\varepsilon_{r}^{\prime}$ value obtained based on measured resonant frequency is 4.23 which is closed to the relative permittivity 4.3. It's clearly that equation (12) is fairly reliable for predicting the dielectric constants of known MUTs based on measured $f_{r . M U T}$. To calculate the relative permittivity of known MUT, equation (12) can be express as:

$$
\varepsilon_{r}^{\prime}=\frac{0.74629-\sqrt{0.55695-0.16608\left(11.575-f_{r . M U T}\right)}}{0.08304}+1
$$

Equation (13) can be used to calculate relative permittivity of known MUTs. In order to check the reliability and validity of the simulation model and (13), the relative permittivity of different MUTs are calculated based on the measured $f_{r . M U T}$ using proposed sensor and are tabulated in Table 6. And Fig. 24 shows the calculated relative permittivity agree well with the literature values. The reliability of calculated $\varepsilon_{r}^{\prime}$ shows that the sensor has the ability to identify different MUTs and predict their dielectric constant within a certain range of accuracy. 


\begin{tabular}{cccc}
\hline \hline Material Under Test (MUT) & Relative Permittivity $\left(\varepsilon_{r}^{\prime}\right)$ & Estimated $\varepsilon_{r}^{\prime}$ based on $(13)$ & Estimation Error \\
\hline Air & 1 & 1.00 & $+0.0 \%$ \\
Teflon & 2.1 & 2.17 & $+3.3 \%$ \\
Quartz & 3.75 & 3.71 & $-1.1 \%$ \\
FR-4 & 4.3 & 4.23 & $-1.6 \%$ \\
Ceramics & 9 & 9.02 & $+0.2 \%$ \\
\hline \hline
\end{tabular}

Table 6. Estimated relative permittivity using measured results and equation (13)

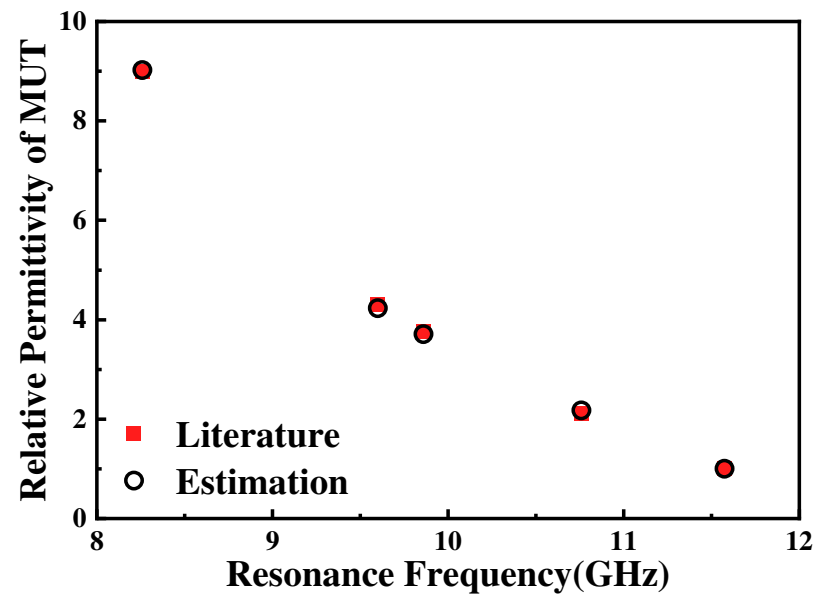

Figure 24. Comparison between the literature values of the relative permittivity from reference ${ }^{9-12}$ and the ones estimated using the transcendental equation (13).

\section{Performance comparison}

The microwave sensor proposed in this paper can be used not only for identifying organic liquids but also for distinguishing solid substrates. So as to place the present work in context, the performance of the proposed sensor is compared with microwave sensors for liquids and microwave sensors for solids reported in the prevailing literature. Moreover, to make a fair comparison of the sensitivity between the proposed sensor and other microwave sensors, we use the mean sensitivity S defined in reference ${ }^{23}$ and shown in (14):

$$
S=\left(\frac{f_{\varepsilon_{r 2}}-f_{\varepsilon_{r 1}}}{f_{0}\left(\varepsilon_{r 2}-\varepsilon_{r 1}\right)}\right) \times 100
$$

Comparison with prevailing sensors for liquids. Table 7 presents the performance characteristics of several conventional microwave sensors with various configurations, resonance frequencies, and excitation sources, etc. Most of conventional meta-atom sensors excited by microstrip line are used for liquids whose permittivity ranges from 9 to 80 . The proposed sensor, excited by a pair of antennas, is designed for liquids with low permittivity which complements the detection range of traditional sensors. Moreover, based on the measured results and mean sensitivity $S$ defined in reference ${ }^{23}$, Table 7 shows that the proposed metamaterial-inspired sensor can distinguish different liquids whose permittivity ranges from 1 to 9 with high mean sensitivity.

\begin{tabular}{|c|c|c|c|c|c|c|}
\hline Ref & Sensor & $\begin{array}{c}f_{\text {res }} \\
{[\mathrm{GHz}]}\end{array}$ & Contact & $\begin{array}{l}\text { Excitation } \\
\text { Source }\end{array}$ & $\begin{array}{c}\text { Permittivity } \\
\text { Range Studied }\end{array}$ & $\begin{array}{l}\text { Mean Sensitivity } S \\
\left(\frac{\boldsymbol{f}_{\varepsilon_{r 2}}-\boldsymbol{f}_{\varepsilon_{r 1}}}{\boldsymbol{f}_{\mathbf{0}}\left(\boldsymbol{\varepsilon}_{r 2}-\boldsymbol{\varepsilon}_{r 1}\right)}\right) \times \mathbf{1 0 0}\end{array}$ \\
\hline [3] & CSRR & 2.4 & NO & $\begin{array}{l}\text { Microstrip } \\
\text { Line }\end{array}$ & $9-80$ & 0.179 \\
\hline [4] & CSRR & 2.1 & NO & $\begin{array}{l}\text { Microstrip } \\
\text { Line }\end{array}$ & $9-80$ & 0.082 \\
\hline [5] & CSRR & 2.4 & YES & $\begin{array}{l}\text { Microstrip } \\
\text { Line }\end{array}$ & $11-60$ & 0.420 \\
\hline [8] & Circular CSRR & 2.4 & NO & $\begin{array}{l}\text { Microstrip } \\
\text { Line }\end{array}$ & $9-79$ & 0.002 \\
\hline [13] & SRR & 3 & NO & Antenna & $13-70$ & 0.035 \\
\hline [23] & MTM Sensor & 2.6 & NO & $\begin{array}{l}\text { Microstrip } \\
\text { Line }\end{array}$ & $1-140$ & 0.27 \\
\hline [24] & $\lambda / 2$ & 2.4 & YES & $\begin{array}{l}\text { Microstrip } \\
\text { Line }\end{array}$ & $7.5-22$ & 0.070 \\
\hline Proposed & $\begin{array}{c}\text { AESRR } \\
\text { Metamaterial }\end{array}$ & 11.5 & YES & Antenna & $1-9$ & 0.612 \\
\hline
\end{tabular}

Table 7. Comparison of the proposed sensor with other microwave sensor for liquids testing 
Comparison with prevailing sensors for solids. Table 8 presents the performance characteristics of several conventional microwave sensors with various configurations, excitation sources, permittivity range studied and frequency shift $\Delta f$, etc. Many conventional meta-atom sensors excited by microstrip line have been reported to be used for distinguishing different dielectric materials and predicting their permittivity. But conventional meta-atom sensors have a lot of room for improvement in terms of frequency shift $\Delta f$ and sensitivity. Moreover, Table 8 shows that the proposed metamaterial-inspired sensor, excited by antenna, can distinguish different solid dielectric materials with bigger frequency shift $\Delta f$ and higher mean sensitivity.

\begin{tabular}{|c|c|c|c|c|c|c|}
\hline Ref & Sensor & $\begin{array}{c}f_{\text {res }} \\
{[\mathrm{GHz}]}\end{array}$ & $\begin{array}{l}\text { Excitation } \\
\text { Source }\end{array}$ & $\begin{array}{c}\text { Permittivity } \\
\text { Range Studied }\end{array}$ & $\begin{array}{c}\text { Frequency Shift } \\
\nabla f(\mathrm{GHz})\end{array}$ & $\begin{array}{l}\text { Mean Sensitivity } S \\
\left(\frac{\boldsymbol{f}_{\varepsilon_{r 2}}-\boldsymbol{f}_{\varepsilon_{r 1}}}{\boldsymbol{f}_{0}\left(\boldsymbol{\varepsilon}_{r 2}-\boldsymbol{\varepsilon}_{r 1}\right)}\right) \times \mathbf{1 0 0}\end{array}$ \\
\hline [9] & CCSR & 2.3 & $\begin{array}{l}\text { Microstrip } \\
\text { Line }\end{array}$ & $1-7$ & 0.48 & 3.45 \\
\hline [10] & SRR & 5.0 & $\begin{array}{l}\text { Microstrip } \\
\text { Line }\end{array}$ & $1-9$ & 0.95 & 2.30 \\
\hline [10] & SRR & 10.0 & $\begin{array}{l}\text { Microstrip } \\
\text { Line }\end{array}$ & $1-9$ & 2.00 & 2.50 \\
\hline [11] & CCSRs & 1.0 & $\begin{array}{l}\text { Microstrip } \\
\text { Line }\end{array}$ & $1-4.3$ & 0.21 & 3.57 \\
\hline [12] & CSRR & 2.7 & $\begin{array}{l}\text { Microstrip } \\
\text { Line }\end{array}$ & $2.2-10.2$ & 0.59 & 2.73 \\
\hline Proposed & $\begin{array}{c}\text { AESRR } \\
\text { Metamaterial }\end{array}$ & 11.5 & Antenna & $1-9$ & 3.32 & 3.59 \\
\hline
\end{tabular}

Table 8. Comparison of the proposed sensor with other microwave sensor for solids testing

\section{Conclusion}

A high-sensitivity microwave metamaterial-inspired sensor, based on a $13 \times 13$ arrays of Asymmetric Electric Split-Ring Resonator (AESRR), is presented for the permittivity characterization of organic liquids and solid dielectric substrates with low permittivity. Excited by a pair of patch antennas, the sensor exhibits strong electric field in the gaps of AESRR which allows the sensor is sensitive to the change of dielectric environment. T-shape channels were integrated to the sensor by grooving in the substrate to improve the integration and enable the feasibility of liquids detection.

During the measurement session, seven organic liquids and four solid dielectric substrates were chosen as MUTs and the measured results match well with the simulated results which verified the reliability of sensor. Based on the fabricated sensor and actual measurement environment, simulation models of measuring liquids and measuring solids were built in CST, respectively. Moreover, two transcendental equations, derived from the measured results, are proposed to predict the relative permittivity of liquid samples and solid materials, respectively. And the estimated values of relative permittivity are in good agreement with the literature values showing the accuracy of transcendental equations.

Compared to prevailing conventional meta-atom microwave sensors excited by microstrip line, the proposed sensor can distinguish not only liquids but also solid dielectric materials with bigger frequency shift $\Delta f$ and higher sensitivity. This sensor has many advantages, such as low-cost, real-time, high-sensitivity, and high-robustness. Most importantly, it applies to the permittivity characterization of organic liquids as well as solid dielectric substrates - a wider range of applications, which makes the sensor an attractive choice to be implemented in a lab-on-a-chip sensor system in the microwave band.

Future work will focus on increasing the sensitivity of sensor and reducing sensor size and reducing the volume/area of MUTs.

\section{References}

1. R.A. Awang, T. Baum, M. Nasabi, S. Sriram, W.S.T. Rowe, Mechanically tolerant fluidic split ring resonators, Smart Mater. Struct. 25 (2016).

2. T. Chen, S. Li, H. Sun, Metamaterials application in sensing, Sensors. 12, 2742-2765 (2012).

3. M. D. Characterization, A. Ebrahimi, S. Member, and W. Withayachumnankul, "High-Sensitivity Metamaterial-Inspired Sensor for Microfluidic Dielectric Characterization," 14, 1345-1351, (2014).

4. W. Withayachumnankul, K. Jaruwongrungsee, A. Tuantranont, C. Fumeaux, and D. Abbott, "Metamaterial-based microfluidic sensor for dielectric characterization," Sensors Actuators, A Phys., 189, 233-237, (2013),

5. X. Zhang, C. Ruan, T. Ul Haq, and K. Chen, "High-sensitivity microwave sensor for liquid characterization using a complementary circular spiral resonator," Sensors (Switzerland), 19, 2019.

6. R. López-Blanco, D. Moreno-González, R. Nortes-Méndez, J. F. García-Reyes, A. Molina-Díaz, and B. Gilbert-López, "Experimental and theoretical determination of pesticide processing factors to model their behavior during virgin olive oil production," Food Chem., 239, 9-16, (2018).

7. W. Cao, R. Singh, I. A. I. Al-Naib, M. He, A. J. Taylor, and W. Zhang, "Low-loss ultra-high-Q dark mode plasmonic Fano metamaterials," Opt. Lett., 37, 3366, (2012).

8. E. L. Chuma, Y. Iano, G. Fontgalland, L. L. B. Roger, and H. Loschi, "PCB-integrated non-destructive microwave sensor for liquid dielectric spectroscopy based on planar metamaterial resonator,” Sensors Actuators, A Phys., 312, (2020), 
9. T. U. Haq, C. Ruan, X. Zhang, and S. Ullah, "Complementary Metamaterial Sensor for Nondestructive Evaluation of Dielectric Substrates," Sensors (Basel)., 19, 1-13, (2019).

10. S. Lim, C. Y. Kim, and S. Hong, "Simultaneous Measurement of Thickness and Permittivity by Means of the Resonant Frequency Fitting of a Microstrip Line Ring Resonator," IEEE Microw. Wirel. Components Lett., 28, 539-541, (2018).

11. M. S. Boybay and O. M. Ramahi, "Material characterization using complementary split-ring resonators," IEEE Trans. Instrum. Meas., 61, 3039-3046, (2012).

12. C. Sen Lee and C. L. Yang, "Complementary split-ring resonators for measuring dielectric constants and loss tangents," IEEE Microw. Wirel. Components Lett., 24, 563-565, (2014).

13. R. A. Awang, F. J. Tovar-Lopez, T. Baum, S. Sriram, and W. S. T. Rowe, "Meta-atom microfluidic sensor for measurement of dielectric properties of liquids," J. Appl. Phys., 121, (2017).

14. V. Sekar, W. J. Torke, S. Palermo, and K. Entesari, "A self-sustained microwave system for dielectric-constant measurement of lossy organic liquids," IEEE Trans. Microw. Theory Tech., 60, 1444-1455, (2012).

15. U. Fano, "Effects of configuration interaction on intensities and phase shifts," Phys. Rev., 124, 1866-1878, (1961).

16. G. Galindo-Romera, F. Javier Herraiz-Martínez, M. Gil, J. J. Martínez-Martínez, and D. Segovia-Vargas, "Submersible Printed Split-Ring Resonator-Based Sensor for Thin-Film Detection and Permittivity Characterization,” IEEE Sens. J.,16, 3587-3596, (2016).

17. S. Rubalya Valantina, D. R. Phebee Angeline, S. Uma, and B. G. Jeya Prakash, "Estimation of dielectric constant of oil solution in the quality analysis of heated vegetable oil," J. Mol. Liq., 238, 136-144, (2017).

18. S. Rubalya Valantina, S. Uma, B. G. Jeya Prakash, D. R. Phebee Angeline, A. Alfred Maxwell, and R. Aravindhan, "Modelling, characterization and quality analysis of heated oil using electric moment and chemical properties," J. Food Sci. Technol., 56, 571-579, (2019).

19. L. Su, J. Mata-Contreras, P. Vélez, A. Fernández-Prieto, and F. Martín, "Analytical method to estimate the complex permittivity of oil samples," Sensors (Switzerland), 18, 1-12, (2018).

20. N. K. Tiwari, S. P. Singh, and M. J. Akhtar, "Novel Improved sensitivity planar microwave probe for adulteration detection in edible oils," IEEE Microw. Wirel. Components Lett., 29, 164-166, (2019).

21. U. Møller, D. G. Cooke, K. Tanaka, and P. U. Jepsen, "Terahertz reflection spectroscopy of Debye relaxation in polar liquids [Invited]," J. Opt. Soc. Am. B, 26, p. A113, (2009).

22. Lim, S., Kim, C. Y., \& Hong, S. Simultaneous Measurement of Thickness and Permittivity by Means of the Resonant Frequency Fitting of a Microstrip Line Ring Resonator. IEEE Microwave and Wireless Components Letters, 28, 539-541. (2018)。

23. M. Abdolrazzaghi, M. Daneshmand, and A. K. Iyer, "Strongly enhanced sensitivity in planar microwave sensors based on metamaterial coupling," arXiv, 66, 1843-1855, (2017).

24. C. Liu and Y. Pu, "A microstrip resonator with slotted ground plane for complex permittivity measurements of liquids," IEEE Microw. Wirel. Components Lett., 18, 257-259, (2008).

25. CST Corp., CST PS Tutorial. [Online]. Available: http//www.cst-china.cn.

26. User Manual, CST Particle Studio SUITE, Darmstadt, Germany, 2012.

27. Y. Cao, K. Chen, C. Ruan, and X. Zhang, "Robust and sensitive metamaterial-inspired microfluidic sensor for liquids with low dielectric constants,” Sensors Actuators, A Phys, 331, (2021).

\section{Acknowledgements}

This work was supported by the National Natural Science Foundation of China (Grant No. 61831001).

\section{Author contributions statement}

Y. Cao proposed the AESRR structure to test different liquids and the method to achieve it, participated in doing experiments and recording the data, cataloged references, analyzed the measured results, and prepared for the whole manuscript; K.Chen participated in the design of the sensor, experiment, and the preparation of the manuscript. K.Chen and Y.Cao contribute equally to this paper; C.Ruan is the research head of experiment, proposed the design and experiment idea, analyzed the data, and participate in the revision of the paper; X.Zhang provided critical feedback and helped shaped the research, analysis and manuscript.

\section{Additional information}

Competing interests: The authors declare no competing interests. 